\title{
Phase behaviour of mixtures of colloidal spheres and excluded-volume polymer chains
}

\author{
D G A L Aarts ${ }^{1}$, R Tuinier ${ }^{2}$ and H N W Lekkerkerker ${ }^{1}$ \\ ${ }^{1}$ Van't Hoff Laboratory, Debye Research Institute, University of Utrecht, Padualaan 8, \\ $3584 \mathrm{CH}$ Utrecht, The Netherlands \\ ${ }^{2}$ Forschungszentrum Jülich, Institut für Festkörperforschung, Weiche Materie, \\ D-52425 Jülich, Germany \\ E-mail: h.n.w.lekkerkerker@chem.uu.nl
}

Received 1 July 2002

Published 9 August 2002

Online at stacks.iop.org/JPhysCM/14/7551

\begin{abstract}
We study the phase behaviour of mixtures of colloidal spheres and polymers that have an excluded-volume interaction dispersed in a (background) solvent using the concept of free volume theory. The depletion layer thickness is calculated from the negative adsorption of polymer segments around a sphere. The correlation length and thermodynamic properties of the excluded-volume interacting polymer chains in solution are taken into account by using results from the renormalization group theory. For small polymer-colloid size ratios the difference from an ideal description of the polymers is small, while for larger size ratios the gas-liquid coexistence region shifts in the direction of higher polymer concentrations and at the same time the liquid-crystal coexistence region becomes more extended. Both the gas-liquid region and the gasliquid-crystal region become less extended. These features are compared to experiment.
\end{abstract}

\section{Introduction}

Mixtures of colloids and non-adsorbing polymers display a rich phase behaviour, involving colloidal 'gas' (poor in colloid, rich in polymer), colloidal 'liquid' (rich in colloid, poor in polymer) and colloidal 'crystal' phases (rich in colloid, poor in polymer). This phase behaviour finds its origin in the interaction between colloidal particles in a sea of polymers. Between two particles the interaction was first described by Asakura and Oosawa [1, 2], Vrij [3] and Joanny et al [4] who showed that there is an osmotic imbalance pushing the particles together if they are within a certain distance of each other. Subsequent calculations for the phase behaviour of colloidal spheres and polymers in a 'background' solvent based on perturbation approaches were performed by Gast et al [5] and Vincent and co-workers [6, 7]. These approaches successfully identified that the topology of the phase diagram depends on the 
polymer-to-colloid size ratio $q=R_{g} / R_{c}$, with $R_{g}$ the polymer's radius of gyration and $R_{c}$ the radius of the colloid. For a concise review of this early work, see [8]. In these approaches the polymer partitioning between coexisting phases was not taken into account. This issue was first addressed by Lekkerkerker [9] and developed in collaboration with Peter Pusey and coworkers using the concept of free volume theory [10]. It is especially successful in explaining why one should find a three-phase coexistence region instead of a three-phase coexistence line. Extensive computer simulations [11-13] and exact solutions in one dimension [1416] validate the free volume approximation. It qualitatively and, for polymers much smaller than the colloids, even quantitatively predicts the correct phase behaviour as can be seen by comparison with experiments done in the laboratory of Peter Pusey [17-21]. The main limitation of the original free volume approach is that it considers the polymers as ideal. Recently, other theoretical approaches to describe colloid-polymer mixtures were explored, aiming at a better description of the polymer [22-25] (for a review, see [26]). In this paper we extend the free volume theory to describe the phase behaviour of mixtures of colloidal spheres and polymer chains with excluded-volume interactions.

In section 2 we will briefly explain the thermodynamic framework needed to calculate phase behaviour using the free volume theory. The theory for ideal polymers and colloidal spheres of Lekkerkerker et al [10] and the resulting phase diagrams will be discussed in section 3. In this approach polymers are modelled as penetrable hard spheres with a radius $R_{g}$. Therefore, the polymers are ideal and the depletion layer thickness around a colloid $\Delta$ is equal to $R_{g}$. However, when using the polymer density profile around a sphere [27, 28] and replacing this profile by a step function, $\Delta$ becomes a function of the curvature $q\left(=R_{g} / R_{c}\right)$ as will also be shown in section 3 . In section 4 we make the transition from ideal polymers to polymers with excluded-volume interactions using results from renormalization group (RG) theory [29]. We will use an expression from Hanke et al [30] to incorporate curvature effects. In section 5 the resulting phase diagrams are presented and compared to the original free volume theory and to experimental phase diagrams. We will summarize and conclude with our findings in section 6.

\section{Free volume theory}

The natural thermodynamic potential to use when calculating the phase behaviour of colloidpolymer mixtures is the semi-grand canonical potential [9]. The colloids are treated canonically, while the polymers are treated grand canonically as is schematically shown in figure 1. The solvent is treated as background. The semi-grand canonical potential $\Omega\left(N, T, V, \mu_{p}\right)$ can be written as

$$
\Omega\left(N, V, T, \mu_{p}\right)=F(N, V, T)-\int_{-\infty}^{\mu_{p}^{r}} N_{p} \mathrm{~d} \mu_{p}^{r^{\prime}},
$$

in which $F(N, V, T)$ is the Helmholtz free energy of a pure hard-sphere dispersion and depends on the number of colloidal particles $N$, the system volume $V$ and the temperature $T$. The reservoir is filled with polymers up to a final chemical potential of polymers $\mu_{p}^{r}$, resulting in $N_{p}$ polymers being pushed into the system. To calculate $N_{p}$ as a function of $\mu_{p}^{r}$ the following assumption is made:

$$
N_{p}\left(\mu_{p}^{r}\right)=n_{p}^{r}\left\langle V_{\text {free }}\right\rangle_{0}=n_{p}^{r} \alpha V
$$

saying that $N_{p}$ is equal to the number density of polymers in the reservoir with $n_{p}^{r}$ times the free volume of the unperturbed system $\left\langle V_{\text {free }}\right\rangle_{0}$. This free volume is equal to the free volume 


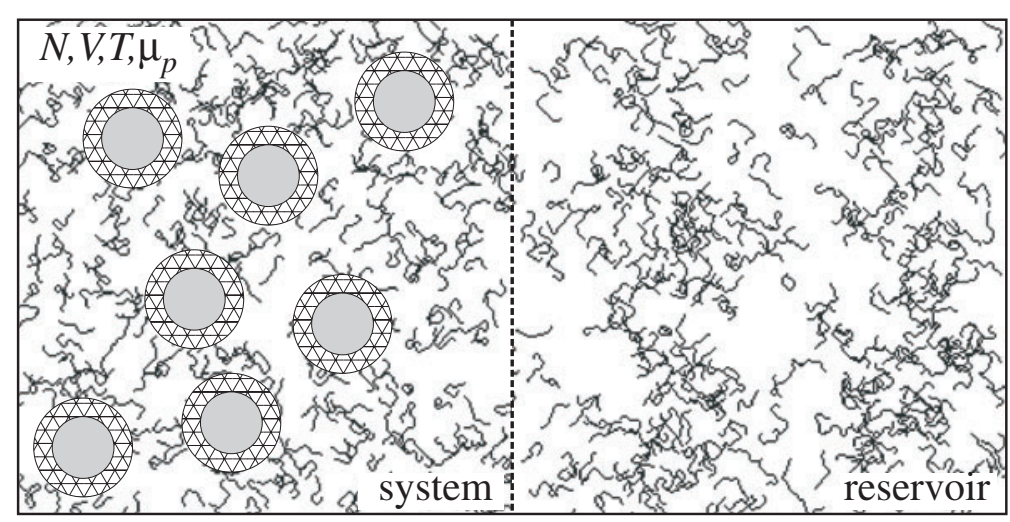

Figure 1. A schematic representation of the semi-grand canonical scheme. The reservoir is filled with polymer and connected to the system via a semi-permeable membrane. The system contains both polymers and colloids and its free volume is the total system volume $V$ minus the volume of the depleted zones. Note that the centres of mass of the polymers cannot penetrate the depletion zones.

fraction $\alpha$ times the system volume $V$. The free volume fraction can be found from scaled particle theory $[10,31]$ and reads

$$
\alpha=\left(1-\phi_{c}\right) \exp \left[-\left(A d+B d^{2}+C d^{3}\right)\right]
$$

with $d=\phi_{c} /\left(1-\phi_{c}\right)$ and the quantities $A, B$ and $C$ functions of the depletion thickness (in this approach a step function with thickness $\Delta$ ) divided by $R_{c}$ :

$$
\begin{aligned}
& A=3 \frac{\Delta}{R_{c}}+3\left(\frac{\Delta}{R_{c}}\right)^{2}+\left(\frac{\Delta}{R_{c}}\right)^{3} \\
& B=\frac{9}{2}\left(\frac{\Delta}{R_{c}}\right)^{2}+2\left(\frac{\Delta}{R_{c}}\right)^{3} \\
& C=3\left(\frac{\Delta}{R_{c}}\right)^{3} .
\end{aligned}
$$

The integration over $\mu_{p}^{r}$ in (2.1) can be replaced with an integration over $n_{p}^{r}$ by applying the Gibbs-Duhem relation:

$$
\mathrm{d} \mu_{p}^{r}=\frac{1}{n_{p}^{r}} \mathrm{~d} \Pi=\frac{1}{n_{p}^{r}}\left(\frac{\partial \Pi}{\partial n_{p}^{r}}\right)_{T} \mathrm{~d} n_{p}^{r} .
$$

Here $\Pi$ is the osmotic pressure of the polymers. Finally, it is convenient to rewrite (2.1) in a dimensionless form:

$$
\tilde{\omega}=\tilde{f}-\int_{0}^{\phi_{p}^{r}} \alpha\left(\frac{\partial \tilde{\Pi}}{\partial \phi_{p}^{r^{\prime}}}\right)_{T} \mathrm{~d} \phi_{p}^{r^{\prime}},
$$

with $\tilde{\omega}=\Omega v_{c} / k_{B} T V$ (with $k_{B}$ the Boltzmann constant and $v_{c}=\frac{4}{3} \pi R_{c}^{3}$ the volume of a colloid), $\tilde{f}=F v_{c} / k_{B} T V$ and $\tilde{\Pi}=\Pi v_{c} / k_{B} T$ the dimensionless osmotic pressure of the polymer solution. Furthermore, $\phi_{p}^{r}$ is the polymer volume fraction $\left(\phi_{p}^{r}=n_{p}^{r} v_{p}\right.$ with $v_{p}=\frac{4}{3} \pi R_{g}^{3}$ ). Equation (2.6) is the central equation in this thermodynamic scheme. The 
coexisting phases follow from the conditions (common-tangent procedure)

$$
\begin{aligned}
& \left(\frac{\partial \tilde{\omega}_{1}}{\partial \phi_{c 1}}\right)_{\phi_{p}^{r}}=\tilde{\mu}_{1}\left(\phi_{p}^{r}\right)=\tilde{\mu}_{2}\left(\phi_{p}^{r}\right)=\left(\frac{\partial \tilde{\omega}_{2}}{\partial \phi_{c 2}}\right)_{\phi_{p}^{r}} \\
& \tilde{\omega}_{1}-\left(\frac{\partial \tilde{\omega}_{1}}{\partial \phi_{c 1}}\right)_{\phi_{p}^{r}} \phi_{c 1}=\tilde{P}_{1}\left(\phi_{p}^{r}\right)=\tilde{P}_{2}\left(\phi_{p}^{r}\right)=\tilde{\omega}_{2}-\left(\frac{\partial \tilde{\omega}_{2}}{\partial \phi_{c 1}}\right)_{\phi_{p}^{r}} \phi_{c 2}
\end{aligned}
$$

Throughout this paper we use a very accurate expression for the free energy of the pure hard-sphere system $\tilde{f}$ following from the Carnahan-Starling equation of state [32]:

$$
\tilde{f}=\phi_{c} \ln \phi_{c}+\frac{4 \phi_{c}^{2}-3 \phi_{c}^{3}}{\left(1-\phi_{c}\right)^{2}}-\phi_{c} .
$$

This can be used to describe both gas $(G)$ and liquid $(L)$ phases as well as the fluid $(F)$ phase. To describe the crystalline phase $(C)$ we make use of a reference free energy obtained from computer simulations [33], from which the crystalline free energy $\tilde{f}_{c}$ follows as

$\tilde{f}_{c}=-\tilde{P}_{c}+\phi_{c} \tilde{\mu}_{c}=-\frac{3 \phi_{c}}{1-\phi_{c} / \phi_{c p}}+\phi_{c}\left(2.1306+\frac{3}{1-\phi_{c} / \phi_{c p}}+3 \ln \left[\frac{\phi_{c}}{1-\phi_{c} / \phi_{c p}}\right]\right)$

with the closest-packed volume fraction $\phi_{c p}=\pi \sqrt{2} / 6$. Except for the thermodynamic properties of the polymer solution and the depletion layer thickness, we have all the ingredients to calculate the binodals using (2.7).

\section{Ideal polymers}

The osmotic pressure of ideal polymers is given by Van't Hoff's law $\Pi=n_{p} k_{B} T$ and the osmotic compressibility $\partial \tilde{\Pi} / \partial \phi_{p}^{r}$ is then simply $1 / q^{3}$. The integral in (2.6) now becomes

$$
\int_{0}^{\phi_{p}^{r}} \alpha\left(\frac{\partial \tilde{\Pi}}{\partial \phi_{p}^{r^{\prime}}}\right)_{T} \mathrm{~d} \phi_{p}^{r^{\prime}}=\frac{\alpha}{q^{3}} \phi_{p}^{r}
$$

If $\Delta$ is known we have all the ingredients for calculating the phase behaviour. Lekkerkerker et al [10] used $\Delta=R_{g}$ and thereby implicitly treated the polymers as penetrable hard spheres with effective radius $R_{g}$. In that case $\Delta / R_{c}$ becomes $q$ in (2.4).

Although the typical length scale of an ideal polymer solution in the bulk is indeed the radius of gyration, the typical length scale near a wall or spherical colloidal surface is different. The depletion thickness near an interface can be calculated if the polymer density profile is known. The density profile is found by solving the Edwards diffusion equation $[34,35]$. Near a single wall, this profile was calculated by Eisenriegler [36], leading to $\Delta=2 R_{g} / \sqrt{\pi}$. The polymer density profile around a sphere was derived by Taniguchi et al [27] and independently by Eisenriegler et al [28]. In the appendix the depletion thickness as a function of curvature is derived for ideal polymers resulting in (A.4)

$$
\frac{\Delta}{R_{c}}=\left(1+\frac{6}{\sqrt{\pi}} q+3 q^{2}\right)^{1 / 3}-1
$$

This expression for $\Delta / R_{c}$ is used in (2.4). In the limit of $q \rightarrow 0, \Delta / R_{c}$ becomes $2 q / \sqrt{\pi}$ agreeing with the flat-wall case and for larger $q, \Delta / R_{c}$ decreases. Curvature effects are now included in the free volume approach. The resulting phase diagrams are presented in figure 2. Clearly, the effect of curvature on the general phase behaviour is small in the ideal case, except for very large $q$ where $\Delta \ll R_{g}$. 
(a)

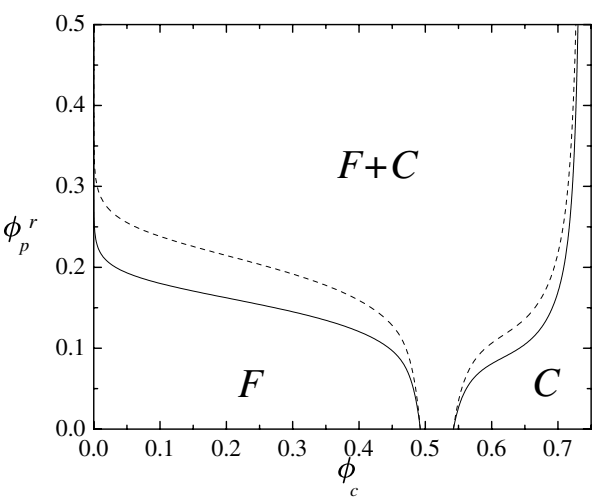

(c)

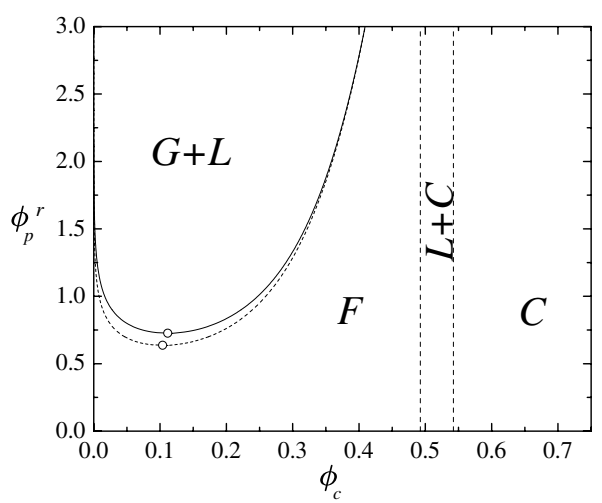

(b)

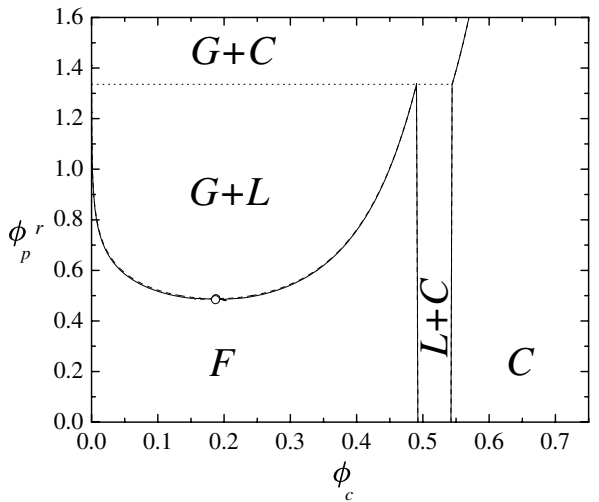

(d)

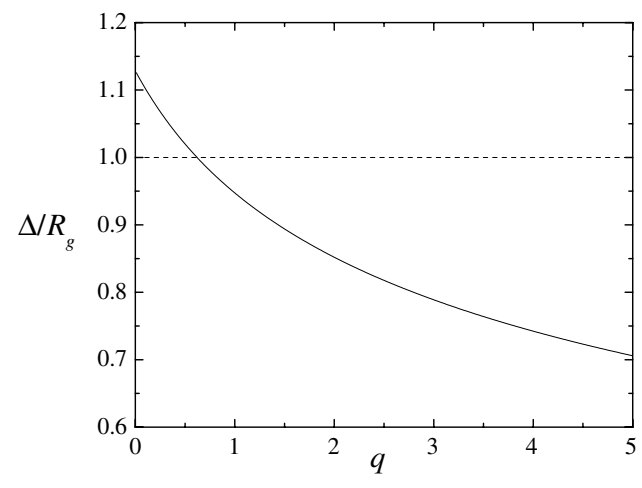

Figure 2. (a)-(c) Phase diagrams for polymer-colloid mixtures with different polymer-colloid size ratios (respectively, $q=0.1,0.6,1.0$ ) in the reservoir concentration. In (d) the curvature dependence of $\Delta / R_{g}$ as in (3.2) is plotted. Dashed curves follow from the theory described in [10] ( $\Delta=R_{g}$ ), while solid curves are the result when using (3.2). Dotted curves denote triple points (gas-liquid-crystal coexistence) and open circles denote critical points. $G, L, F$ and $C$ denote colloidal gas, liquid, fluid and crystal phases respectively.

\section{Polymers with excluded-volume interactions}

In good solvents excluded-volume interactions between polymer segments have to be taken into account in order to describe the thermodynamics of the polymers in solution properly. Thus, we first need an expression for the osmotic compressibility. This can be found from RG theory [29] for polymers in the full excluded-volume limit and is in dimensionless form equal to

$$
\left(\frac{\partial \tilde{\Pi}}{\partial \phi_{p}^{r}}\right)=\frac{1}{q^{3}}\left(1+2.629 \phi_{p}^{r}\left(\frac{1+3.251 \phi_{p}^{r}+4.151\left(\phi_{p}^{r}\right)^{2}}{1+1.480 \phi_{p}^{r}}\right)^{0.309}\right) .
$$

Secondly, curvature effects have to be incorporated. Hanke et al [30] calculated the insertion energy to place one colloidal sphere in a sea of excluded-volume polymers. This energy is equal to the total number of polymers depleted from a certain region and is analysed in terms of flat and curvature terms. We can calculate a depletion thickness from this number by making 
(a)

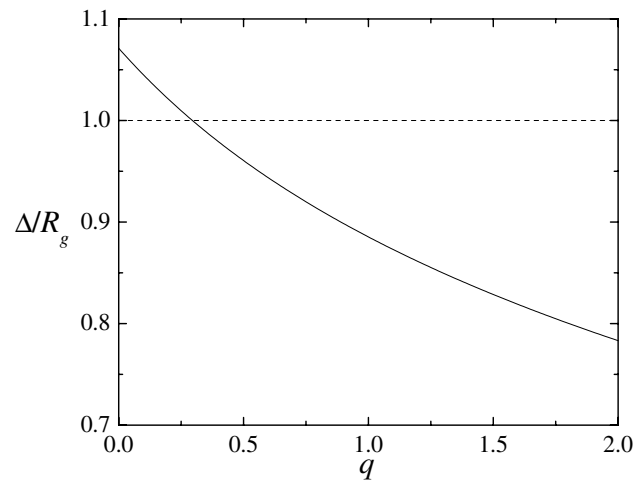

(b)

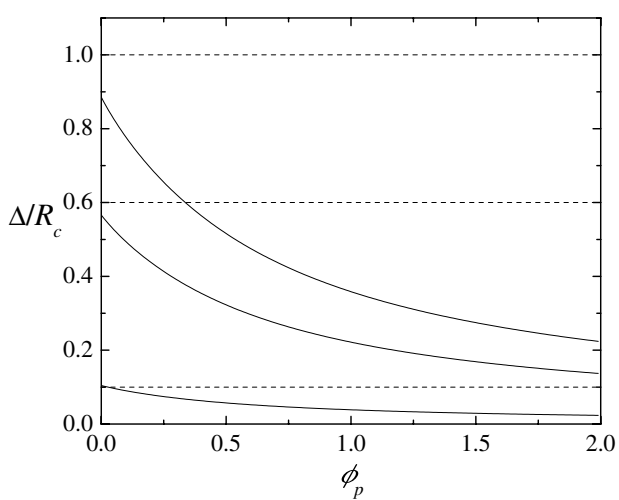

Figure 3. (a) Curvature dependence of $\Delta / R_{g}$ and (b) concentration dependence of $\Delta / R_{C}$ following (4.2) (full curves) with modifications explained in the text and for the ideal case with $\Delta=R_{g}$ (dashed lines). The concentration dependence is shown for three different polymer-colloid size ratios; from top to bottom curves: $q=1.0,0.6$ and 0.1 .

use of a step function and $\Delta / R_{C}$ becomes

$$
\frac{\Delta}{R_{c}}=\left(1+3 a\left(\frac{R_{g}}{R_{c}}\right)+3 b\left(\frac{R_{g}}{R_{c}}\right)^{2}-3 c\left(\frac{R_{g}}{R_{c}}\right)^{3}\right)^{1 / 3}-1,
$$

with

$$
\begin{aligned}
& a=\frac{2}{\sqrt{\pi}}\left[1-\frac{1}{4}\left(1-\frac{3}{2} \ln 2-\frac{\pi}{2}+\frac{\pi}{\sqrt{3}}\right)\right] \approx 1.0710 \\
& b=1-\frac{5 \pi}{8}+\frac{17}{36}+\frac{\pi \sqrt{3}}{4} \approx 0.8691 \\
& c=\frac{1}{3 \sqrt{\pi}}\left(\frac{1673 \pi}{48}-\frac{551}{15}-\frac{40 \pi}{\sqrt{3}}\right) \approx 0.0399 .
\end{aligned}
$$

Note that (4.2) is the RG theory result up to third order in curvature and hence applicable for small $q$. To incorporate the polymer concentration dependence we replace $R_{g}$ with the correlation length in bulk which is in line with the work of Joanny et al [4]. Expressions for the bulk correlation length as well as the osmotic compressibility are taken from [29] and we have all the ingredients for (2.6). In figure 3(a) we show the curvature dependence of $\Delta / R_{g}$, which clearly deviates from the standard ideal case with $\Delta=R_{g}$ and in figure 3(b) the concentration dependence of $\Delta / R_{c}$ is plotted. The depletion thickness decreases strongly as a function of the polymer concentration.

\section{Results}

In this section we present phase diagrams obtained with the proposed theory for mixtures of colloidal hard spheres and polymer chains with excluded-volume interactions between the segments in a common solvent. The results are compared with those for mixtures of ideal polymers mixed with colloidal spheres as described in [10] and with experimental phase diagrams. In figure 4 we present phase diagrams for three different polymer-colloid size ratios. For $q=0.1$ (figure $4(\mathrm{a})$ ) the difference between describing the polymers as ideal 
(a)

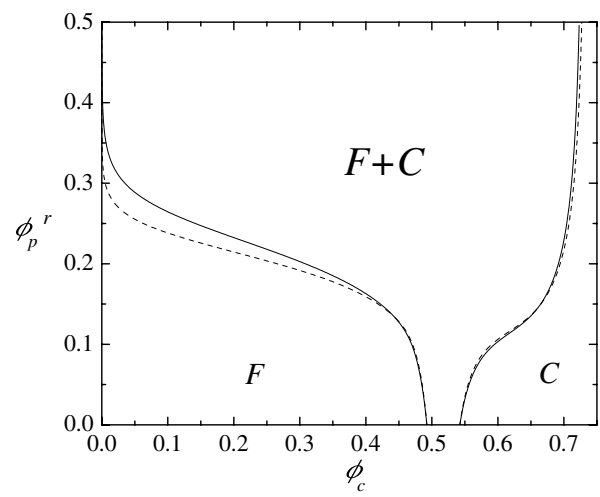

(c)

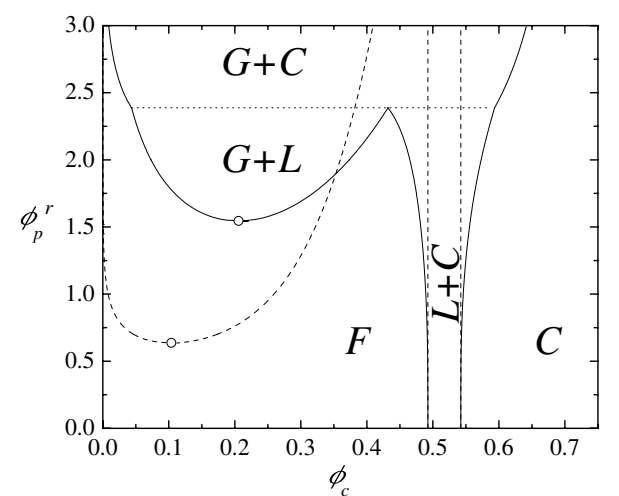

(b)

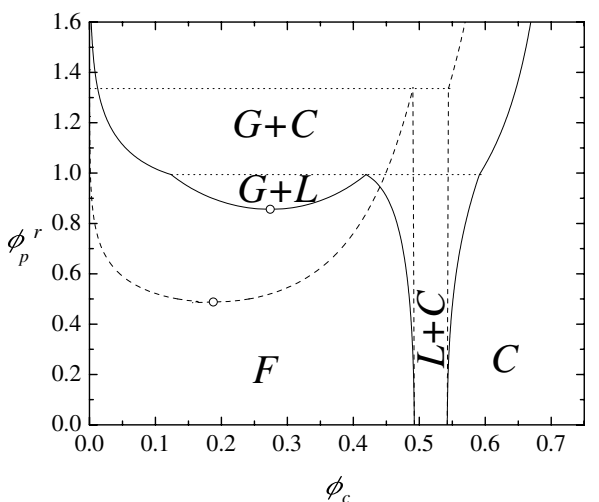

(d)

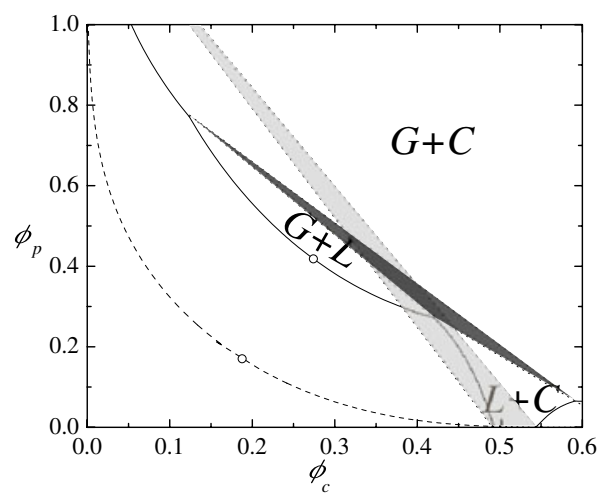

Figure 4. (a)-(c) Phase diagrams for polymer-colloid mixtures with different polymer-colloid size ratios (respectively, $q=0.1,0.6,1.0$ ) in reservoir concentration. Dashed curves follow from the theory described in [10] ( $\Delta=R_{g}$ ), while solid curves are valid for mixtures of excluded-volume polymers and colloids and follow from (4.2) with modifications explained in the text. (d) The phase diagram in the system polymer concentration for $q=0.6$. The light area is the three-phase region for ideal polymers and the dark area that for excluded-volume polymers. Dotted curves denote triple points (gas-liquid-crystal coexistence) and open circles depict critical points.

following [10] or with excluded-volume interactions is clearly very small, while the difference is much larger for $q=0.6$ and 1.0 (figures 4 (b) and (c)). Since the depletion thickness $\Delta$ becomes smaller than $R_{g}$ if $q>0.30$ (figure 3(a)) and because $\Delta$ decreases rapidly as a function of polymer concentration (figure 3(b)), more polymer is needed for gas-liquid phase separation to occur for $q=0.6$ and 1.0. Furthermore, the gas-liquid region becomes quickly metastable with respect to gas-crystal coexistence due to the strongly rising osmotic compressibility (4.1) compared to the ideal case. Related to this, gas-liquid coexistence first becomes stable for $q=0.48$, while this is at $q=0.33$ for ideal polymers. In figure 4(d) we make the transition to actual polymer concentrations for $q=0.6$ by multiplying the reservoir polymer volume fraction $\phi_{p}^{r}$ with the free volume fraction in one phase $\alpha\left(\phi_{c 1}\right): \phi_{p 1}=\phi_{p}^{r} \alpha\left(\phi_{c 1}\right)$. Here, we see that the liquid-crystal region is much larger than in the ideal case. In addition, the three-phase coexistence region is shifted upwards and becomes slightly smaller.

In figure 5 we make the comparison with experimental phase diagrams. Ilett et al constructed phase diagrams for mixtures of polymethylmethacrylate (PMMA) colloidal 
(a)

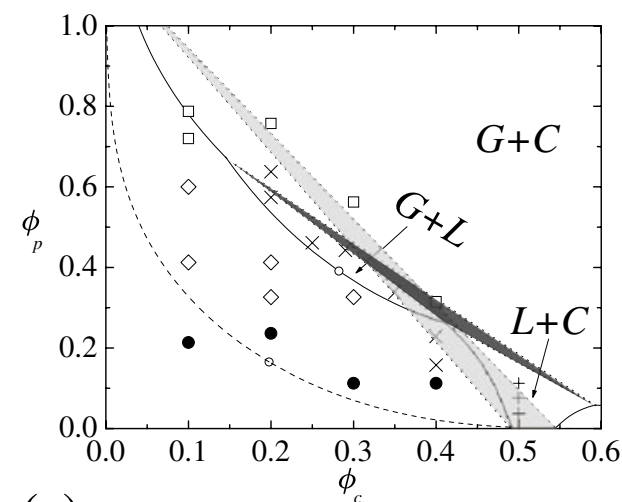

(c)

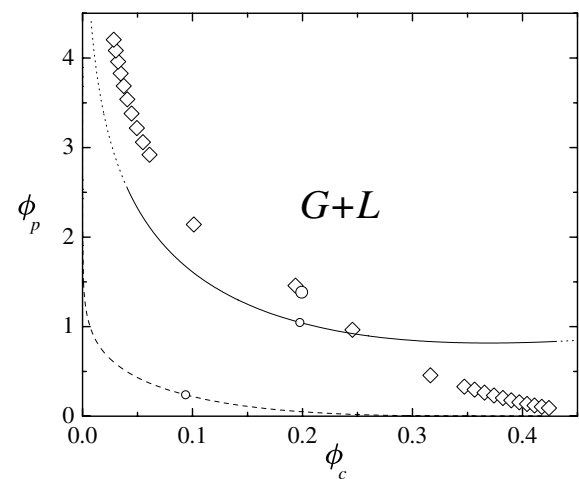

(b)

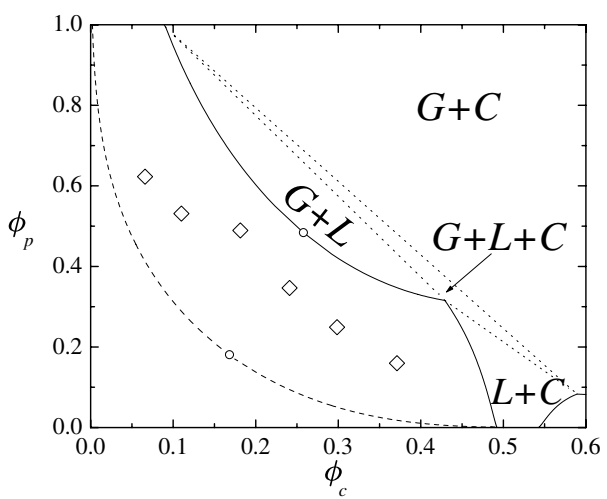

Figure 5. Comparison of experiment with the ideal theory described in [10] (dashed curves) and the model proposed here for excluded-volume polymers (full curves) with experimental phase diagrams for (a) $q=0.57$ from [19], (b) $q=0.667$ from [37] and (c) $q=1.08$ from [38]. The open circles denote critical points, while the open diamonds are the experimental binodal points in (b) and (c). The symbols in (a) denote the following: filled circles: fluid; diamonds: gas plus liquid; crosses: gas plus liquid plus crystal; plus signs: liquid plus crystal; squares: gas plus crystal. The light area is the three-phase region for ideal polymers and the dark area that for excluded-volume polymers.

spheres and polystyrene polymer [19]. In figure 5(a) we compare their constructed phase diagram for $R_{c}=228 \mathrm{~nm}$ and $R_{g}=130 \mathrm{~nm}(q=0.57)$ with the original free volume theory and with the extension described in section 4 to include excluded-volume polymers. Although the gas-liquid region is underestimated by the extended theory, both the liquid-crystal as well as the three-phase region are much better predicted. More recently, Ramakrishnan et al [37] measured the binodal for mixtures of colloidal silica spheres coated with stearyl alcohol $\left(R_{c}=50 \mathrm{~nm}\right)$ and polystyrene polymer in toluene. Here, we compare the result with polystyrene with a molar mass of $5.5 \times 10^{5} \mathrm{~g} \mathrm{~mol}^{-1}\left(R_{g}=33.37 \mathrm{~nm}\right)$ resulting in a size ratio $q=0.667$ in figure $5(\mathrm{~b})$. The open diamonds are the experimental points. The original theory clearly predicts polymer concentrations that are too low at the binodal while the present theory (full line) overpredicts the required polymer concentration at the binodal. In figure 5(c) we compare with the experimental phase line of mixtures of small stearyl-coated 
silica spheres $\left(R_{c}=13 \mathrm{~nm}\right)$ and polydimethylsiloxane polymer $\left(R_{g}=14 \mathrm{~nm}\right)$ in cyclohexane with a size ratio $q=1.08$. De Hoog and Lekkerkerker [38] measured the binodal in great detail by following the method of Bodnar and Oosterbaan [39]. The dashed line is the ideal polymer theory result and again the new theory (full curve) predicts phase separation to occur at much higher polymer concentration. This full curve is partially dotted where the gas-liquid phase separation is no longer stable with respect to gas-crystal phase separation. However, no gas-crystal phase coexistence was observed experimentally possibly due to suppression by sphere polydispersity which is not accounted for in the present theory. Furthermore, the present theory predicts too high polymer concentration above a certain colloid volume fraction, the reason for which is unknown.

\section{Discussion and conclusions}

The effects of curvature and of excluded-volume polymers on the phase behaviour of colloidpolymer mixtures have been taken into account by extending the free volume theory [10]. For ideal polymers, curvature effects are included by using the polymer density profile [27, 28], which has a small effect on the general phase behaviour. For excluded-volume polymers results from RG theory $[29,30]$ were used and in that case both the description with excluded-volume polymers and the curvature play a role. For small size ratios (as shown with $q=0.1$ ) the depletion thickness is approximately $R_{g}$; curvature effects are weak and the concentration is not yet sufficiently large to decrease the correlation length significantly with respect to $R_{g}$. For $q=0.6$ and 1.0 the curvature effects are stronger and in particular the correlation length has dropped significantly at the polymer concentrations where the system becomes unstable. Thus, the gas-liquid phase coexistence will shift to larger polymer concentrations for excludedvolume polymers as compared to ideal polymers. At the same time the liquid-crystal region becomes larger while the three-phase coexistence region becomes less extended.

In comparison with experiment we see that the ideal polymer theory predicts too low polymer concentrations for gas-liquid phase separation to occur and that our new model predicts too high polymer concentrations, except for $q=1.08$ [38]. Although the phase diagram for this colloid-polymer mixture was carefully determined, both colloidal spheres and polymers are rather polydisperse, the effects of which are unclear. Liquid-crystal coexistence, clearly present in experiment for $q=0.57$, is almost absent in the ideal polymer theory (a discrepancy of about a factor of 250 [21]). A significantly more accurate description of the liquid-crystal coexistence region is perhaps one of the major achievements of the present theory. We conclude that modifying the free volume theory to incorporate excludedvolume polymers is rather straightforward and results in a better description of some of the characteristic features in the experimental phase diagrams. More sophisticated theories are, however, required to obtain a more complete quantitative agreement with experiments.

\section{Appendix. Depletion thickness for ideal polymers}

Placing a colloidal sphere in a sea of polymers depletes $N_{p}^{d e p}$ polymers from a certain volume. This number is equal to the polymer number density $n_{p}$ times the volume that is depleted of polymers, which is the colloid volume $v_{c}$ plus a region around the colloid found from the polymer concentration profile $f(x)=n(x) / n_{b}$ :

$$
N_{p}^{d e p}=n_{p} v_{c}+n_{p} \int_{R_{c}}^{\infty} 4 \pi r^{2}(1-f(x)) \mathrm{d} x .
$$


The profile $[27,28]$ gives the local polymer segment density $n(x)$ at a distance $x$ from the colloidal surface divided by the polymer bulk segment density $n_{b}$ and has the following form:

$$
\begin{aligned}
f(x)=\left[\frac{R_{c}}{R_{c}+x}\right]^{2}\left[\left(\frac{x}{R_{c}}\right)^{2}+\frac{2 x}{R_{c}}\left(\operatorname{erf}(z)-2 z^{2}[1-\operatorname{erf}(z)]+\frac{2}{\sqrt{\pi}} z \exp \left(-z^{2}\right)\right)\right. \\
+2 \operatorname{erf}(z)-\operatorname{erf}(2 z)+\frac{4 z}{\sqrt{\pi}}\left[\exp \left(-z^{2}\right)-\exp \left(-4 z^{2}\right)\right] \\
\left.+8 z^{2}\left[\frac{1}{2}-\operatorname{erf}(2 z)+\frac{1}{2} \operatorname{erf}(z)\right]\right],
\end{aligned}
$$

where $z=x / 2 R_{g}$. This polymer profile can be replaced by a step function and doing so defines a depletion thickness $\Delta$, which can be found by solving (A.3) for $\Delta$ :

$$
n_{p} v_{c}+n_{p} \int_{R_{c}}^{\infty} 4 \pi r^{2}(1-f(x)) \mathrm{d} x=n_{p} \frac{4 \pi}{3}\left(R_{c}+\Delta\right)^{3} .
$$

The resulting curvature dependence of $\Delta(q)$ becomes after doing the integration

$$
\frac{\Delta}{R_{c}}=\left(1+\frac{6 q}{\sqrt{\pi}}+3 q^{2}\right)^{1 / 3}-1
$$

\section{References}

[1] Asakura S and Oosawa F 1954 J. Chem. Phys. 221255

[2] Asakura S and Oosawa F 1958 J. Polym. Sci. 33183

[3] Vrij A 1976 Pure Appl. Chem. 48471

[4] Joanny J F, Leibler L and De Gennes P G 1979 J. Polym. Sci.: Polym. Phys. 171073

[5] Gast A P, Hall C K and Russel W B 1983 J. Colloid Interface Sci. 96 251-67

[6] Vincent B 1987 Colloids Surf. 24 269-82

[7] Vincent B, Edwards J, Emmett S and Croot R 1988 Colloids Surf. 31 267-98

[8] Pusey P N 1989 Colloidal Suspensions. Les Houches: Liquides, Cristallisation et Transition Vitreuse/Liquids, Freezing and Glass Transition (Amsterdam: Elsevier)

[9] Lekkerkerker H N W 1990 Colloids Surf. 51 419-26

[10] Lekkerkerker H N W, Poon W C K, Pusey P N, Stroobants A and Warren P B 1992 Europhys. Lett. 20 559-64

[11] Meijer E J and Frenkel D 1994 J. Chem. Phys. $1006873-87$

[12] Bolhuis P and Frenkel D 1994 J. Chem. Phys. 101 9869-75

[13] Dijkstra M, Brader J M and Evans R 1999 J. Phys.: Condens. Matter 11 10079-106

[14] Lekkerkerker H N W and Widom B 2000 Physica A 285 483-92

[15] Brader J M and Evans R 2002 Physica A 306287

[16] Oversteegen S M and Lekkerkerker H N W 2002 Physica A 310 181-96

[17] Pusey P N, Pirie A D and Poon W C K 1993 Physica A 201 322-31

[18] Pusey P N, Poon W C K, Ilett S M and Bartlett P 1994 J. Phys.: Condens. Matter 6 A29-36

[19] Ilett S M, Orrock A, Poon W C K and Pusey P N 1995 Phys. Rev. E 51 1344-52

[20] Poon W C K, Pirie A D and Pusey P N 1995 Faraday Discuss. 101 65-76

[21] Moussaïd A, Poon W C K, Pusey P N and Soliva M F 1999 Phys. Rev. Lett. 82 225-8

[22] Louis A A, Bolhuis P G, Hansen J P and Meijer E J 2000 Phys. Rev. Lett. 85 2522-5

[23] Bolhuis P G, Louis A A, Hansen J P and Meijer E J 2001 J. Chem. Phys. 114 4296-311

[24] Bolhuis P G, Louis A A and Hansen J P 2001 Phys. Rev. E 640221801

[25] Bolhuis P G and Louis A A 2002 Macromolecules 35 1860-9

[26] Fuchs M and Schweizer K S 2002 J. Phys.: Condens. Matter 14 R239-69

[27] Taniguchi T, Kawakatsu T and Kawasaki K 1992 Slow Dynamics in Condensed Matter (AIP Series vol 256) (New York: American Institute of Physics) p 503

[28] Eisenriegler E, Hanke A and Dietrich S 1996 Phys. Rev. E 54 1134-52

[29] Schäfer L 1999 Excluded Volume Effects in Polymer Solutions (Heidelberg: Springer)

[30] Hanke A, Eisenriegler E and Dietrich S 1999 Phys. Rev. E 59 6853-78

[31] Reiss H 1992 J. Phys. Chem. 96 4736-47

[32] Carnahan N F and Starling K E 1969 J. Phys. Chem. 51635 
[33] Frenkel D and Ladd A J C 1984 J. Chem. Phys. 813188

[34] Edwards S F and Freed K F 1969 J. Phys. A: Math. Gen. 2145

[35] Grosberg A Y and Khoklov A R 1994 Statistical Mechanics of Macromolecules (New York: American Institute of Physics)

[36] Eisenriegler E 1983 J. Chem. Phys. 791052

[37] Ramakrishnan S, Fuchs M, Schweizer K S and Zukoski C F 2002 J. Chem. Phys. 116 2201-12

[38] de Hoog E H A and Lekkerkerker H N W 1999 J. Phys. Chem. B 103 5274-9

[39] Bodnar I and Oosterbaan W D 1997 J. Chem. Phys. $1067777-80$ 